Hong-Il Im

Nikola Vladimir

Šime Malenica

Dae-Seung Cho

https://doi.org/10.21278/TOF.41304

ISSN 1333-1124

eISSN 1849-1391

\title{
QUASI-STATIC RESPONSE OF A 19,000 TEU CLASS ULTRA LARGE CONTAINER SHIP WITH A NOVEL MOBILE DECKHOUSE FOR MAXIMIZING CARGO CAPACITY
}

\begin{abstract}
Summary
Modern sea transportation is characterized by ever larger container ships, which require direct calculation procedures and numerical tools to achieve their reliable structural design. There are different attempts to increase the ship loading capacity, as for instance a container ship with a novel mobile deckhouse enabling her to carry additional number of containers, and at the same time changing basic structural properties of the ship. This paper is related to structural design evaluation of a 19,000 TEU ultra large container ship with novel mobile deckhouse for maximizing cargo capacity, particularly relying on quasi-static approach. The ship structural design is evaluated both for fatigue and extreme loads, where conventional container ship of same particulars is used as a reference. Independent analyses of new and conventional design indicate that conventional container ship shows somewhat better performance from viewpoint of fatigue, while global extreme loading is at the same level.
\end{abstract}

Key words: $\quad$ Container ship, Spectral fatigue, Extreme response, Quasi-static approach.

\section{Introduction}

The trend in modern sea transportation is building ever larger Container Ships (CS); there are already several vessels in service with a maximum capacity of over 19,000 TEU. A specific characteristic of Ultra Large Container Ships (ULCS), compared with the other ship types, is that they are more likely to experience the hydroelastic type of structural responses called springing and whipping [1], [2], [3], [4], [5], [6], [7]. That is caused by their large dimensions leading to higher structure flexibility, a relatively high service speed, and large bow flare. Apart from that, there are different attempts to improve ship design and to increase the ship's loading capacity; one of them is an ULCS with a mobile deckhouse and an open hold below [8], [9], [10]. The mobile deckhouse enables the ship to carry additional number of containers, but at the same time it slightly changes the original structural design, with additional hatch openings making the vessel more vulnerable to warping deformation, compared with the conventional CS design. 
The existing rules of classification societies cover only a limited size and types of structures, and are mainly based on a quasi-static approach, while high frequency hydroelastic contributions are either neglected or included only empirically [11]. Due to the above mentioned reasons, such solutions do not seem to be entirely appropriate for ULCSs; therefore, direct calculations are needed to ensure their safe and rational design. In this sense, some classification societies have developed guidelines (rule notes) for the inclusion of hydroleastic effects into the overall design procedure [11], [12], [13], [14], associated with the application of relevant hydro-structure software offered around the world. Usually, these tools have different numerical schemes incorporated, but mainly rely on the same theoretical principles. However, there are some difficulties in the application of hydro-structure software, because its validity domain is not fully clear, and maybe, more important, the methodologies of different classification societies are not completely harmonized in the selection of realistic operational profile and statistical post-processing of the signals, both for fatigue and extreme loads.

This paper deals with the evaluation of structural design of a 19,000 TEU ULCS having a novel open hold under a mobile deckhouse. The paper illustrates the application of direct calculation methodologies in the design of ULCSs, concerning only the quasi-static structural response. Two container ships are analysed, i.e. the above mentioned ULCS with a mobile deckhouse and a conventional ULCS having the same main dimensions. Fatigue damage obtained by spectral approach and long-term extreme vertical bending moment (VBM) are selected as relevant quantities for comparison.

The analysis was performed by means of a general hydro-structure tool, HOMER [1], [15], where the general FE code NASTRAN [16] and the potential flow code Hydrostar [17] are used as the structural and the hydrodynamic solver, respectively. It is necessary to mention that the relative importance of hydroelasticity is considered in another paper [18] where the BV Rule Note 583 [13] was followed. The calculation setups for quasi-static calculations and for hydroelastic calculations, [18], are the same; direct comparisons of the relevant quantities and an accurate assessment of the hydroelastic effects are made possible by both setups.

\section{Mobile deckhouse concept and ship particulars}

ULCSs with a capacity of over 10,000 TEU normally adopt the 2-island type superstructure in which a deckhouse structure for accommodation is located in the ship's mid body and is separated from the other funnel and engine casing structure, Figure 1, [8], [9], [10]. Figure 2a shows an arrangement of compartments and spaces around the accommodation in the conventional container ship design. The shaded areas between the upper deck and bridge wings are generally unused spaces and can be considered for extra loading. The space under the upper deck, except the parts for fuel oil tanks and the operation of machinery and facilities, is also available for additional loading. These facts motivated the development of a ship with a mobile deckhouse with the arrangement of compartments shown in Figure 2b. The bench-shaped structure located in the uppermost position shows a mobile part of accommodation, Figure 3. The two structures on both sides used to support the mobile part are named as side towers. Side towers share the function of accommodation together with the mobile part. To arrange a cargo space for an additional 40ft-bay inside the hull structure, the fuel oil tanks with the size of about $40 \mathrm{ft}$ in length, which are located under the accommodation, are rearranged and separated into two parts. Each of the tanks has two 40ftbays in length and is higher than those in the traditional design to ensure the equivalent tank volume. This concept features a bridge and three upper decks mounted on rails, having a capability to move longitudinally. By using the void beneath the sliding block for additional 
storage, as well as by resizing and repositioning fuel tanks, room is created for an additional two $20 \mathrm{ft}$ container bays. Finally, this concept gives an extended loading capacity of around 270, 350, and 450 TEU in 11,000, 14,000, and 19,000 TEU class CSs, respectively, compared with the conventional CS design. The main particulars of the analysed 19,000 TEU container ships are presented in Table 1.

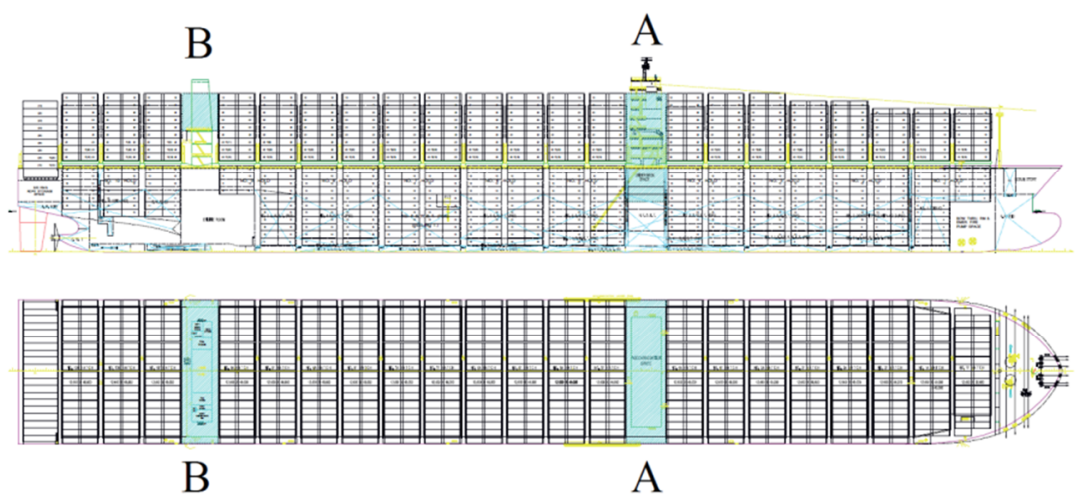

Fig. 1 General arrangement of a 2-island type container carrier [8]

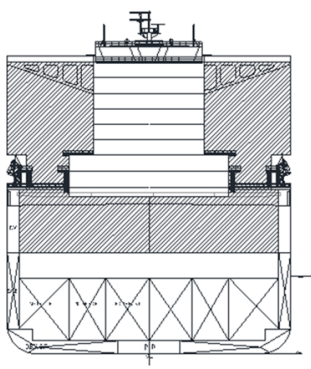

a) Conventional CS

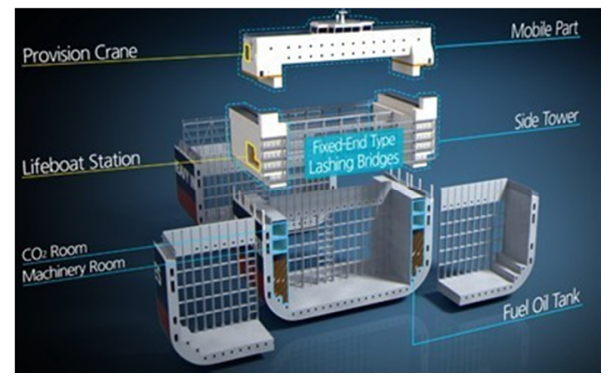

b) CS with a mobile deckhouse

Fig. 2 Arrangement of compartments in the conventional CS design and in the ship with a mobile deckhouse

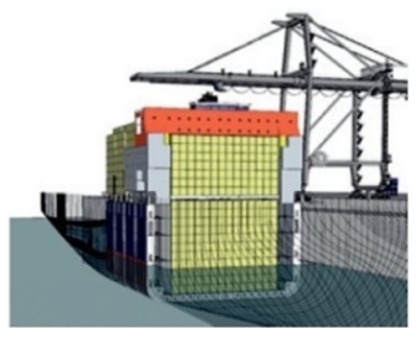

a) Partial view on the ship

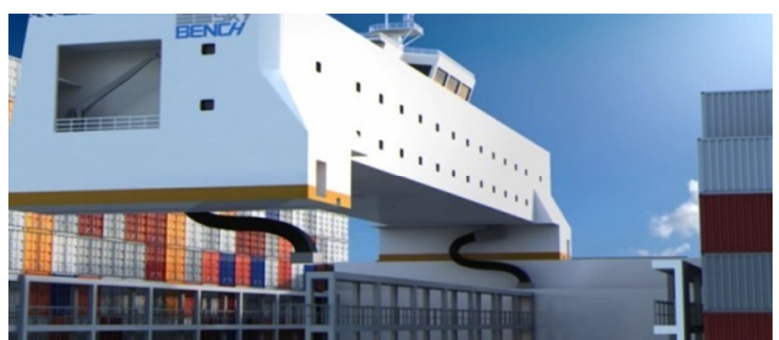

b) Mobile deckhouse

Fig. 3 Illustration of the mobile deckhouse concept with additional holds

Table 1 Main particulars of the analysed container ships

\begin{tabular}{|l|c|}
\hline Length over all, $L_{O A}[\mathrm{~m}]$ & 400 \\
\hline Length between perpendiculars, $L_{P P}[\mathrm{~m}]$ & 383 \\
\hline Breadth, $B[\mathrm{~m}]$ & 58.6 \\
\hline Depth, $H[\mathrm{~m}]$ & 30.5 \\
\hline Design draught, $T_{d}[\mathrm{~m}]$ & 14.5 \\
\hline Scantling draught, $T_{s}[\mathrm{~m}]$ & 16.0 \\
\hline Displacement at full load, $\Delta_{F}[\mathrm{t}]$ & 212913 \\
\hline Service speed, $v_{s}[\mathrm{kn}]$ & 23.0 \\
\hline
\end{tabular}




\section{Description of the assessment procedure}

Reference is made to the newly introduced BV Rule Note NR 583 [13] and to the paper of Malenica and Derbanne [3]. In these documents, the basic assumptions on the numerical methods and the corresponding methodologies are described and are strictly applied in this study. In order to identify the most critical aspects of the approach, we recall below the main assumptions and the calculation steps. For that purpose, different aspects of the hydrostructure interactions are summarized in Table 2.

Table 2 Different hydro-structural issues ( $\mathrm{H}$ - hydrodynamics, $\mathrm{S}$ - structure)

\begin{tabular}{|c|c|c|c|}
\hline$S$ & Linear & $\begin{array}{c}\text { Weakly } \\
\text { nonlinear }\end{array}$ & $\begin{array}{c}\text { Impulsive } \\
\text { nonlinear }\end{array}$ \\
\hline Quasi static & $X$ & $X$ & $X$ \\
\hline Dynamic & $X$ & $X$ & $X$ \\
\hline
\end{tabular}

In order to clear up any misunderstanding in the definition of the quasi-static and dynamic (or hydroelastic) structural responses, we point out here that the quasi-static response is defined as the response in which the ship structural vibrations are not taken into account. Basically, this means that the structural loading comes exclusively from the dynamic pressure associated with the rigid body motions. This hydrodynamic pressure is counter balanced by the ship rigid body inertia which allows for a proper definition of the final quasi-static loading cases.

\subsection{Fatigue assessment}

A classical Palmgren Miner summation approach is adopted. Due to the linearity assumptions, calculations are performed in the frequency domain and the spectral approach is used, which allows for a very low computational cost. The overall computational scheme is shown in Figure 4.

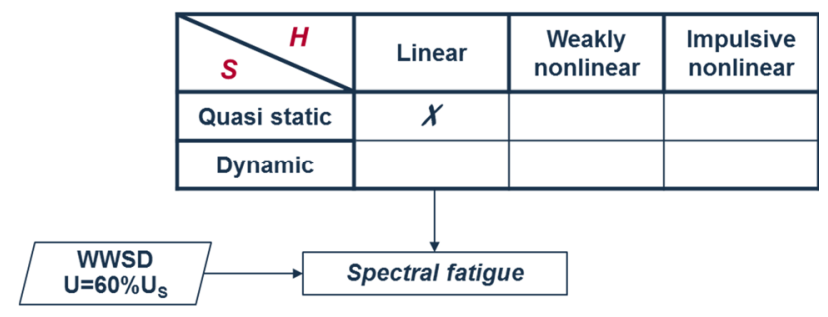

Fig. 4 Spectral fatigue within the quasi-static approach

(WWSD - World Wide Scatter Diagram, U - Ship speed, US - Ship service speed)

\subsubsection{Linear quasi static hydro-structure interaction model in the frequency domain}

According to the quasi-static assumptions, the hydrodynamic and structural calculations can be performed separately. This means that the ship motions are first calculated and they are then used to produce the consistent loading on the FE model. The ship motion equation looks as follows:

$$
\left\{-\omega^{2}([\boldsymbol{M}]+[\boldsymbol{A}(\omega)])-i \omega[\boldsymbol{B}(\omega)]+[\boldsymbol{C}]\right\}\{\xi\}=\left\{\boldsymbol{F}^{D I}(\omega)\right\}
$$

where:
$[M] \quad$ genuine mass matrix of the body
$[\boldsymbol{A}(\omega)] \quad$ hydrodynamic added mass matrix
$[\boldsymbol{B}(\omega)] \quad$ hydrodynamic damping matrix 
$[C] \quad$ hydrostatic restoring matrix

$\left\{\boldsymbol{F}^{D I}(\omega)\right\} \quad$ hydrodynamic excitation vector

$\{\xi\} \quad$ body motions vector (with respect to the ship's centre of gravity $\left(X_{G}, Y_{G}\right.$, $\left.\left.Z_{G}\right)\right)$

Once the ship motions are calculated, the final loading case of the FE model is composed of 3 parts: rigid body inertial loading $\left(-\omega^{2} m_{i} \xi_{i}\right) \quad$, pressure loading $\left(p^{h s 1}+\right.$ $\left.p^{h d}\right)$, and gravity term $\left(-m_{i} g \boldsymbol{\Omega} \times \boldsymbol{k}\right)$.

The last part of the loading comes from the fact that the hydrodynamic and structural calculations are not performed in the same coordinate system (structure in the body fixed and hydrodynamics in the initial earth fixed coordinate system). Thus, a consistent linearization procedure implies the occurrence of the term without which the FE model would not be properly balanced.

The pressure component of the loading is composed of two relatively distinct parts. The first part of the pressure loading is related to the variation of the hydrostatic pressure due to ship motions; it can be written in the following form:

$$
p^{h s 1}=-\varrho g\left[\xi_{3}+\xi_{4}\left(Y-Y_{G}\right)+\xi_{5}\left(X-X_{G}\right)\right]
$$

where $\left(X_{G}, Y_{G}\right)$ are the coordinates of the ship centre of gravity. The second part of the pressure is related to the wave body interactions; it is composed of 8 distinct components (incident, diffracted, and six radiated components):

$$
p^{h d}=p_{I}+p_{D}-i \omega \sum_{j=1}^{6} \xi_{j} p_{R j}
$$

These pressure components are calculated using the potential flow seakeeping theory where the pressure is calculated using the Bernoulli equation which relates the hydrodynamic pressure to the velocity potential. The velocity potential is calculated using the Boundary Integral Equation Method (BIEM). In addition, the general numerical hydrodynamic code Hydrostar is employed in the present study [17].

It is important to notice that, due to the fact that the hydrodynamic and structural calculations are performed separately using different meshes, the final loading case for the FE structural model might experience significant misbalance if the pressure transfer is not done properly. Special attention should be paid to this issue and the way in which this was done in the hydro-structure tool HOMER is described in [3]. Meanwhile, in order to evaluate the local stress concentration in the particular structural detail, the so called top-down analysis should be used [19]. This means that the global structural response is first calculated on a full coarse mesh ship model and the resulting information (deformations, pressure, inertia...) are transferred to the local structural model which is finally solved using the same numerical FE method as the one used for the global response.

\subsubsection{Spectral fatigue}

The stress response amplitude operators (RAO) for all possible combinations of the frequencies and headings should be calculated for spectral fatigue analysis. In practice, 60 frequencies and 36 headings (every 10 degrees) are used. According to the rules, the ship speed is taken to be $60 \%$ of the nominal speed. Once the RAOs are evaluated, they should be combined with the probability of occurrence of different sea states for a given scatter diagram. According to the BV Rules, the scatter diagram which should be used is the so called World Wide Scatter Diagram (WWSD). 


\subsection{Extreme structural response within the quasi-static approach}

Within the verification process for extreme conditions, there are two types of verifications which should be done:

- Yielding \& buckling

- Ultimate strength

\subsubsection{Yielding and buckling}

In the case of yielding and buckling, the procedure requires the determination of several design load cases which are applied on the 3D FE model; for each of them, the yielding and the buckling check is performed.

The computational scheme for the assessment of the extreme structural response within the quasi static approach is shown in Figure 5.

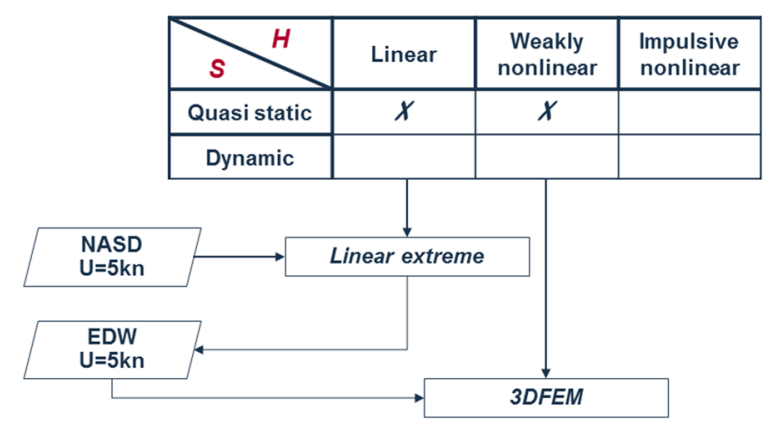

Fig. 5 Extreme structural response within the quasi-static approach (NASD - North Atlantic Scatter Diagram, EDW - Equivalent Design Wave)

The procedure starts by the evaluation of the linear long term values of different Dominant Loading Parameters (DLP) such as vertical bending moment, torsional moment, shear forces, etc. Once the long term value of a particular DLP has been evaluated, it is possible to define the corresponding Equivalent Design Wave (EDW). There are different possibilities to define the EDW, starting from a very simple regular wave and ending with relatively complex irregular response conditioned waves. In the simplest case of the regular unidirectional design wave, the frequency and the wave heading are defined as the ones where the RAO of the considered DLP reaches its maximum and the corresponding design wave amplitude is obtained by dividing the linear long term value by the same RAO. In the case of the irregular response conditioned wave, which is recommended to be used, [20], because it accounts better for the nonlinear hydrodynamic loading, the wave is defined in the form of the design wave spectra with different amplitudes and phases chosen to produce the maximum long term value of the DLP at one particular time instant. We do not go here into the details of the procedure and we refer the reader to [20]. Once the equivalent design wave is determined, it should be applied on the structural FE model of the ship, in a weakly nonlinear sense.

There are different types of the weakly nonlinear models and the one which is used within the Bureau Veritas is the hydro-structure tool HOMER [1]. The method is based on using the linear frequency domain results and transferring them into the time domain where the nonlinear hydrodynamic loading is added at each time step. The time domain motion equation is written in the following form:

$$
\begin{aligned}
\left([\mathbf{m}]+\left[\mathbf{A}^{\infty}\right]\right) & \{\ddot{\xi}(t)\}+([\mathbf{k}]+[\mathbf{C}])\{\xi(t)\}+[\mathbf{b}]\{\dot{\xi}(t)\}+\int_{0}^{t}[\mathbf{K}(t-\tau)]\{\dot{\xi}(\tau)\} d \tau \\
& =\{\mathbf{F}(t)\}+\{\mathbf{Q}(t)\}
\end{aligned}
$$


where $[\mathbf{m}]$ represents the modal mass matrix, $\left[\mathbf{A}^{\infty}\right]$ is the infinite frequency modal added mass matrix, $[\mathbf{k}]$ is the structural stiffness matrix, $[\mathbf{C}]$ represents the hydrostatic restoring matrix, $[\mathbf{b}]$ is the structural damping matrix, $[\mathbf{K}(t-\tau)]$ is the impulse response (memory) functions matrix, while $\{\mathbf{F}(t)\},\{\mathbf{Q}(t)\}$ and $\{\xi(t)\}$ represent the linear excitation force vector, the nonlinear excitation force vector, and the body motions/deformations vector, respectively.

It can be shown that the impulse response functions can be calculated from the linear frequency dependent damping coefficients using the following relation:

$$
\boldsymbol{K}(t)=\frac{2}{\pi} \int_{0}^{\infty} \boldsymbol{B}(\omega) \sin \omega t d \omega
$$

where $B(\omega)$ are the frequency domain damping coefficients, Eq. (1).

Once the impulse response function matrix $[\mathbf{K}(t)]$ is calculated, the motion equation (4) is integrated in time and the nonlinear forces are added at each time step to the vector $\{\mathbf{Q}(t)\}$. There are various types of non-linearities which have to be included in the hydrodynamic model and it is not practically possible to account for all of them. Within the present weakly non-linear hydrodynamic model, the main nonlinearities which are included are related to the so-called Froude Krylov nonlinear loading which accounts for large body motions. In order to illustrate the difference between the linear and the weakly nonlinear hydrodynamic model, the instantaneous pressure distributions on the ship hull for both cases are shown in Figure 6. As it can be seen, the linear pressure "stops" at the mean waterline $Z=0$, while the non-linear pressure extends up to the wave surface.
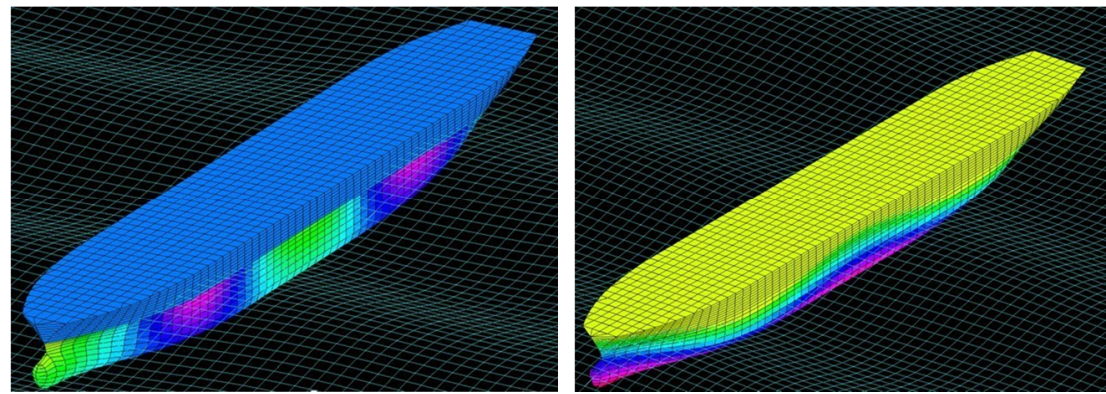

Fig. 6 Hydrodynamic pressure correction close to the waterline (Froude Krylov approximation) and the difference between the linear (left) and the nonlinear (right) pressure distribution.

\subsubsection{Ultimate strength}

According to the current rules, the verification of the ultimate strength requirements is made by comparing the life time maximum bending moment with the structural capacity of each ship section [20]. For the time being, the life time maximum of the vertical bending moment is prescribed by the rules so that there is no need for direct calculations. Since the main objective was to compare the structural response of two ship types within the direct calculation approach, no detailed ultimate strength check was made for this quasi-static part of the analysis and the comparisons were made for WhiSp2 notation only [18].

\section{Results and discussion}

\subsection{Numerical model}

The ship speed is set at $5.0 \mathrm{kn}$ and $13.8 \mathrm{kn}$ (60\% of the design speed) for the extreme and the fatigue response assessment, respectively, [13]. The values of the wave heading angle are considered uniformly distributed from $0^{\circ}$ to $350^{\circ}$ with steps of $10.0^{\circ}$. The analysis is performed by means of a general hydro-structure code which requires the definition of the structural and the hydrodynamic model, as well as the integration mesh aimed for pressure 
H.I. Im, N. Vladimir, Š. Malenica, D.S. Cho
Quasi-Static Response of a 19,000 TEU Class

Ultra Large Container Ship with a Novel Mobile Deckhouse for Maximizing Cargo Capacity

transfer from the hydrodynamic to the structural mesh. 3D FE models of the CS with a mobile deckhouse and of the conventional one are shown in Figure 7, together with indicated fine mesh positions for the fatigue life/damage evaluation. For each of the considered ships, 12 structural details of interest, having the same position along the ship, are chosen for the fatigue damage assessment. The hydrodynamic mesh with nearly 6,000 elements and the integration mesh are the same for both ships; they are presented in Figures 8 and 9, respectively.

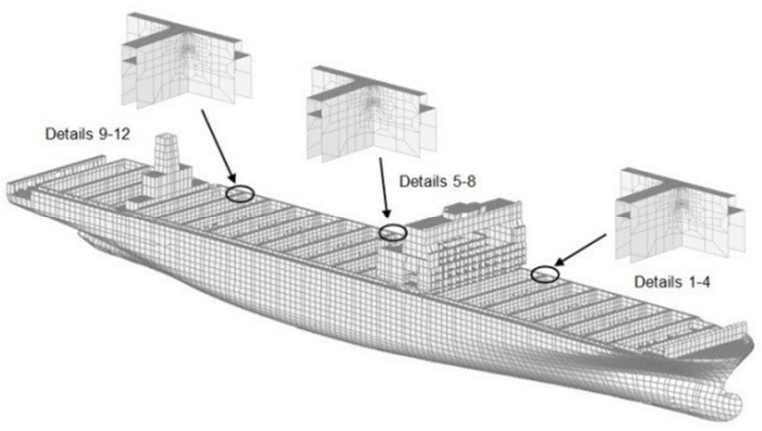

a) CS with a mobile deckhouse

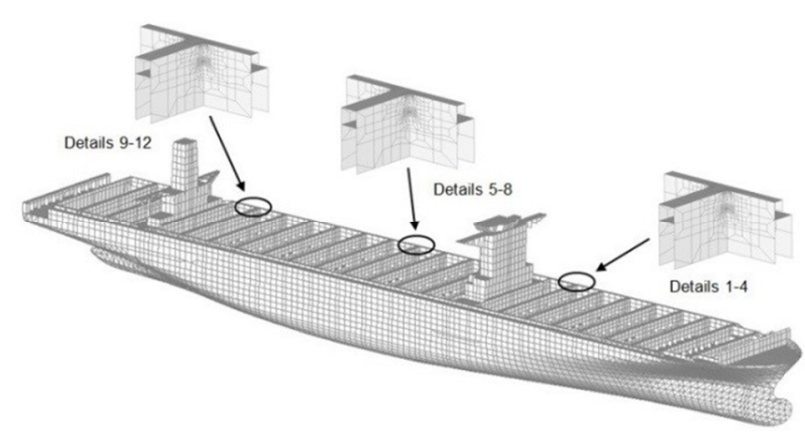

b) Conventional CS

Fig. 7 3D FE models of the analysed container ships with local fine mesh models

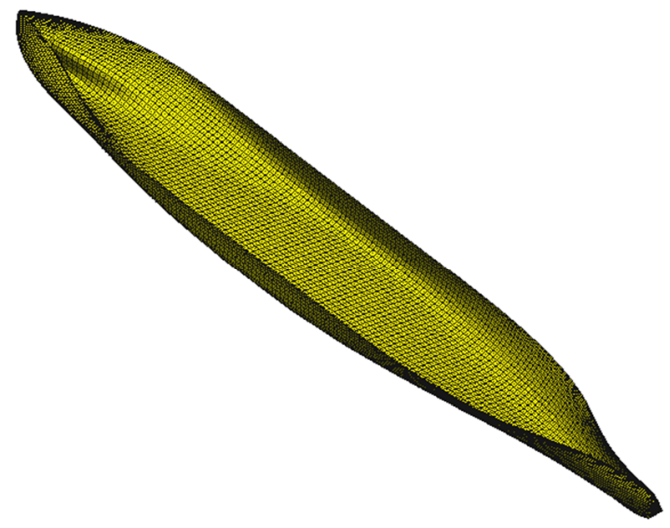

Fig. 8 Hydrodynamic mesh

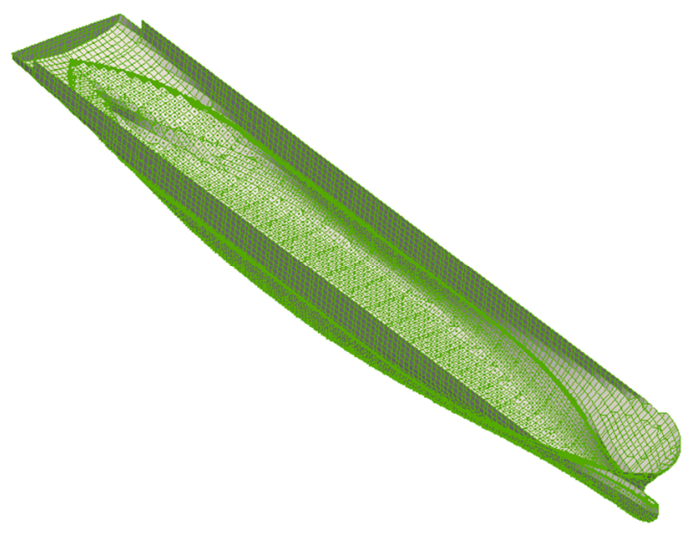

Fig. 9 Integration mesh

In hydro-structure analysis, it is to be checked that the structural model is adequately balanced; this is done by comparing sectional forces and moments with those listed in the loading manual, Fig. 10, where good agreement can be noticed.

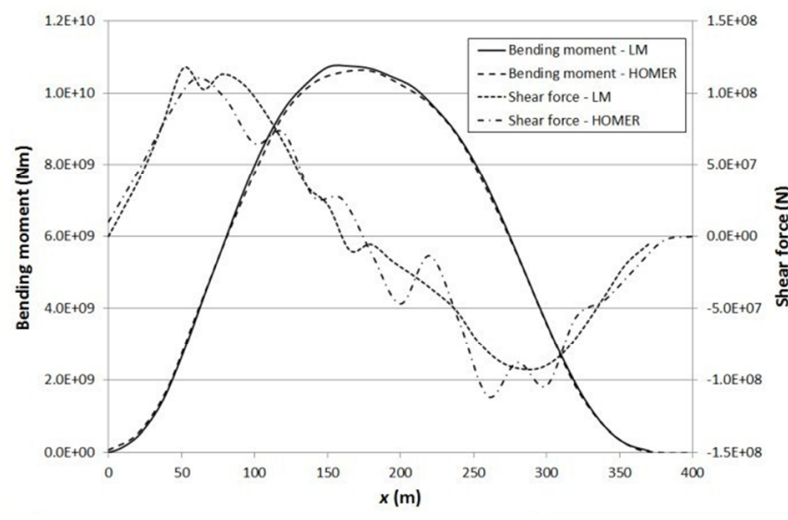

a) CS with a mobile deckhouse

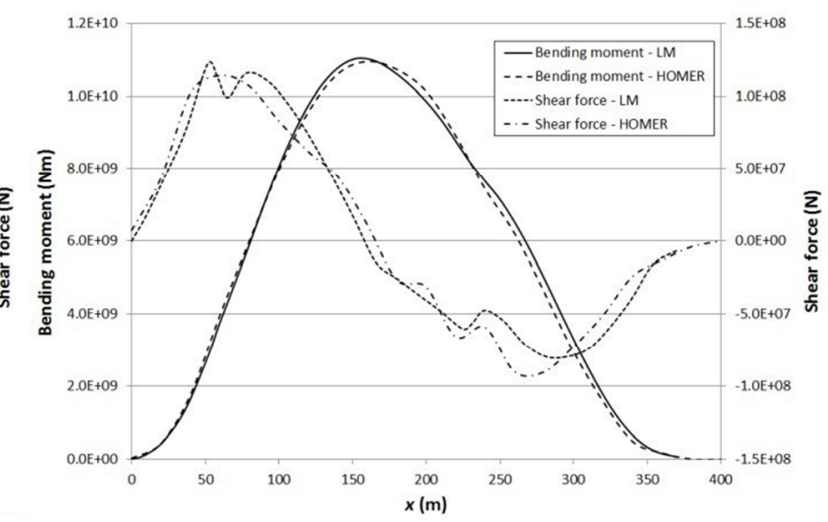

b) Conventional $\mathrm{CS}$

Fig. 10 Still water bending moment and shear forces 


\subsection{Spectral fatigue}

Within the top-down approach, the global response is calculated by coarse mesh 3D FE models, Figure 7, and in the second step, the obtained global response is imposed at the boundaries of fine mesh models to calculate the local stress RAOs, with a typical case shown in Figure 11.

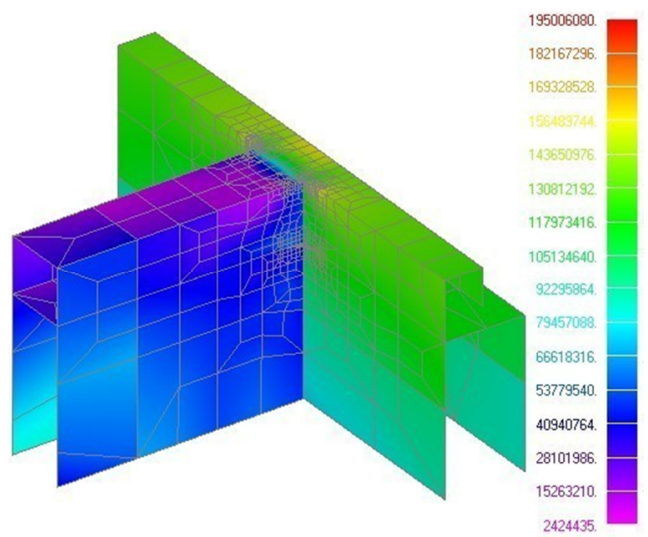

a) Von Mises stresses $(\mathrm{Pa})$

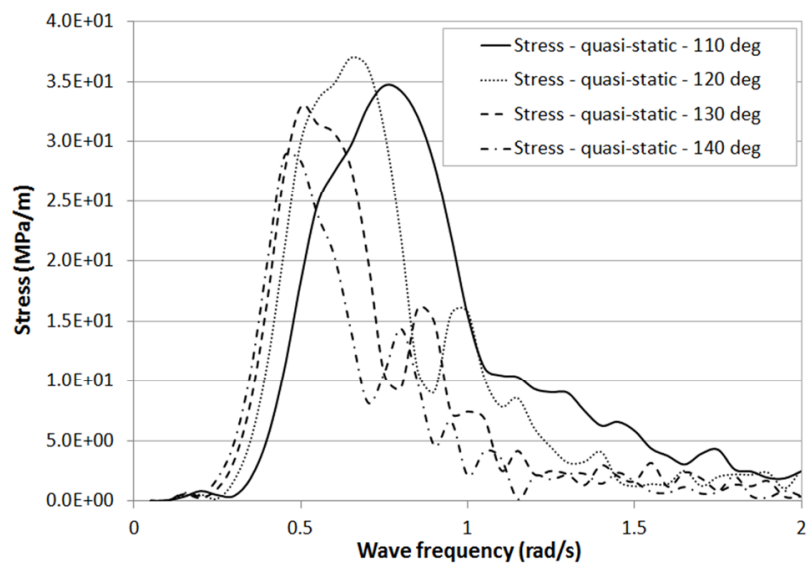

b) Stress RAO sample

Fig. 11 Typical case of von Mises stresses in the fine mesh model and the stress RAO of the container ship with a mobile deckhouse

Stress RAOs are used as input for the fatigue calculation. The axial stress in the rod elements at the hatch corner radius free edges of fine mesh FE models is taken into account. Fatigue damage ratios of the selected structural details are presented in Table 3. The results are obtained for a sailing factor equal to 0.85 and the mean stress effect is taken into account.

Generally, the conventional CS shows somewhat better performance from the viewpoint of fatigue. This is most probably due to the "softer" hull girder characteristics in torsion of the new design, which leads to a slight increase in the local stress concentration close to the critical structural locations. The greatest fatigue damages are obtained for the hatch corners in the vicinity of the engine room area, which is actually typical of CSs.

Table 3 Fatigue damage ratio of the selected structural details obtained by the quasi-static approach

\begin{tabular}{|c|c|}
\hline $\begin{array}{c}\text { Detail } \\
\text { Position }\end{array}$ & $\begin{array}{c}\text { Damage ratio } \\
\text { Conventional CS/Mobile deckhouse CS }\end{array}$ \\
\hline 1 & 1.46 \\
\hline 2 & 0.60 \\
\hline 3 & 1.25 \\
\hline 4 & 0.76 \\
\hline 5 & 1.00 \\
\hline 6 & 0.87 \\
\hline 7 & 0.86 \\
\hline 8 & 0.63 \\
\hline 9 & 0.84 \\
\hline 10 & 0.58 \\
\hline 11 & 0.74 \\
\hline 12 & 0.96 \\
\hline
\end{tabular}




\subsection{Extreme structural response: yielding and buckling}

Within the present approach only the regular EDWs were evaluated. The different values of these EDWs for the vertical bending moment (VBM), the horizontal bending moment (HBM), and the torsional moments (TM) are given in Table 4 for both ships. As one can see, the values for both ships are quite close as expected because the approach is purely quasi-static.

The structural verifications for yielding and buckling were performed for both ships and no critical parts of the structure were identified. This means that both ships are safe from that point of view. Atypical snapshot of structural stresses is shown in Figure 12 for the EDW of the VBM.

Table 4 Values of EDW for the container ship with a mobile deckhouse and the conventional container ship

\begin{tabular}{|c|c|c|c|c|c|c|}
\hline \multirow{2}{*}{ Loading parameter } & \multicolumn{6}{|c|}{ Equivalent Design Wave } \\
\cline { 2 - 7 } & \multicolumn{2}{|c|}{ CS with a mobile deckhouse } & \multicolumn{3}{c|}{ Conventional CS } \\
\cline { 2 - 7 } & $\begin{array}{c}\text { Amplitude } \\
(\mathrm{m})\end{array}$ & $\begin{array}{c}\text { Heading } \\
(\mathrm{deg})\end{array}$ & $\begin{array}{c}\text { Frequency } \\
(\mathrm{rad} / \mathrm{s})\end{array}$ & $\begin{array}{c}\text { Amplitude } \\
(\mathrm{m})\end{array}$ & $\begin{array}{c}\text { Heading } \\
(\mathrm{deg})\end{array}$ & $\begin{array}{c}\text { Frequency } \\
(\mathrm{rad} / \mathrm{s})\end{array}$ \\
\hline VBM amidships & 9.54 & 180.0 & 0.45 & 9.63 & 180.0 & 0.45 \\
\hline HBM amidships & 6.49 & 120.0 & 0.65 & 6.44 & 120.0 & 0.65 \\
\hline TM at 0.25L & 6.38 & 240.0 & 0.65 & 6.29 & 240.0 & 0.65 \\
\hline TM at 0.75 L & 8.16 & 290.0 & 0.65 & 7.64 & 300.0 & 0.65 \\
\hline
\end{tabular}
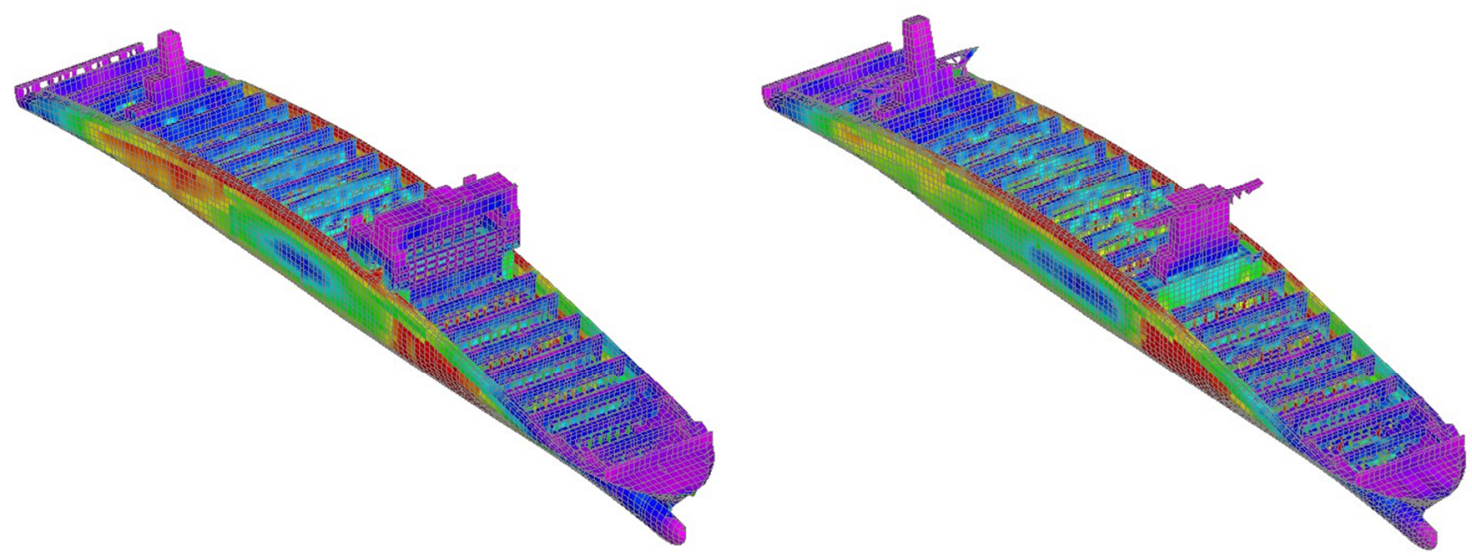

Fig. 12 A typical snapshot of the structural stresses of the container ship with a mobile deckhouse and the conventional container ship for the EDW of the VBM in hogging conditions.

\section{Conclusions}

In order to evaluate the structural design of a newly developed container ship with an open hold under the mobile deckhouse, an analysis of the two container ships of the same principal dimensions is done using the quasi-static approach. The BV Rule Note NR 625 is followed in the definition of the calculation setup [20], and the general hydro-structure code HOMER is applied. Effect of structural modification on the ship performance in waves is checked by comparing the fatigue damage of several structural details and the long term vertical bending moment value of both ships. Fatigue is assessed by the linear spectral analysis, where the top-down procedure is applied. The obtained results indicate that two ships are safe from both fatigue and extreme response points of view. Both ships have similar safety levels in the case of extreme response, while, in the case of fatigue, the conventional 
container ship shows slightly better performance; this is most likely due to an increase in the stress concentration because of the softer torsional behaviour of the hull girder in the case of the new design. However, a more detailed analysis with hydroelastic effects (springing and whipping) included is necessary for a more realistic comparison of two structural designs, which is the subject of future research.

\section{Acknowledgments}

This study was done within the Joint Research Project "A Study on Springing and Whipping of Hyundai SkyBench ${ }^{\mathrm{TM}}$ Container Carrier". The authors would also like to acknowledge the support from the National Research Foundation of Korea (NRF) grant funded by the Korean Government (MSIP) through GCRC-SOP (Grant No. 2011-0030013). The obtained results and conclusions reflect personal opinions of the authors and do not represent the official attitude of their institutions. The authors would also like to express their gratitude to Alexis Benhamou from the Bureau Veritas Research Department for his valuable help.

\section{REFERENCES}

[1] Š. Malenica, Q. Derbanne, F.X. Sireta, F. Bigot, E. Tiphine, G. De Hauteclocque, X.B. Chen: HOMER Integrated hydro-structure interactions tool for naval and offshore applications, International Conference on Computers Applications in Shipbuilding, Busan, Korea, 2013.

[2] Š. Malenica, I. Senjanović, N. Vladimir: Hydro structural issues in the design of ultra large container ships, Brodogradnja, 64(3), 323-347, 2013.

[3] Š. Malenica, Q. Derbanne: Hydro structural issues in the design of ultra large container ships, International Journal of Naval Architecture and Ocean Engineering, 6, 983-999, 2014. https://doi.org/10.2478/IJNAOE-2013-0226

[4] J.H. Kim, Y.H., Kim: Numerical analysis on springing and whipping using fully-coupled FSI models. Ocean Engineering, 91, 28-50, 2014. https://doi.org/10.1016/j.oceaneng.2014.08.001

[5] M. Barhoumi, G. Storhaug: Assessment of whipping and springing on a large container vessel, International Journal of Naval Architecture and Ocean Engineering, 6(4), 442-458, 2014. https://doi.org/10.2478/IJNAOE-2013-0191

[6] I. Senjanović, N. Vladimir, M. Tomić, N. Hadžić, Š. Malenica: Global hydroelastic analysis of ultra large container ships by improved beam structural model, International Journal of Naval Architecture and Ocean Engineering, 6(4), 1041-1063, 2014. https://doi.org/10.2478/IJNAOE-2013-0230

[7] I. Senjanović, N. Vladimir, M. Tomić, N. Hadžić, Š. Malenica: Some aspects of structural modelling and restoring stiffness in hydroelastic analysis of large container ships, Ships and Offshore Structures, 9(2), 199-217, 2014. https://doi.org/10.1080/17445302.2012.762728

[8] H.I. Im, D.S. Cho, T.J. Kim, B.K. Choi, H.R. Ryu, B.R. Lee: A new design of container carrier for maximizing cargo capacity. Proceedings of International Conference Design and Operation of Container Ships, London, UK, RINA, 53-60, 2014.

[9] H.I. Im, D.S. Cho, G.H. Hwang, D.H. Lee, B.K. Choi, B.R. Lee: SkyBench ${ }^{\mathrm{TM}}$ container carriers for maximizing cargo capacity, 28th Asia Pacific Technical Exchange and Advisory Meeting on Marine Structures - TEAM, Istanbul, Turkey, 508-513, 2014.

[10] H.I. Im, N. Vladimir, Š. Malenica, H.R. Ryu, D.S. Cho: Fatigue analysis of HHI SkyBench ${ }^{\mathrm{TM}} 19000$ TEU ultra large container ship with springing effect included, Proceedings of $7^{\text {th }}$ International Conference on Hydroelasticity in Marine Technology, Split, Croatia, 561-574, 2015.

[11] DNV GL: Class Guideline - DNVGL-CG-0153: Fatigue and ultimate strength assessment of container ships including springing and whipping, 2015.

[12] Lloyd's Register: ShipRight design and construction, guidance notes on the assessment of global design loads of large container ships and other ships prone to whipping and springing, London, UK, 2014.

[13] Bureau Veritas: Whipping and Springing Assessment, Rule Note NR 583 DT R00 E. Paris, France, 2015.

[14] American Bureau of Shipping: Whipping assessment for container carriers, American Bureau of Shipping, Houston, TX, USA, 2010. 
H.I. Im, N. Vladimir,

S̆. Malenica, D.S. Cho
Quasi-Static Response of a 19,000 TEU Class

Ultra Large Container Ship with a Novel Mobile Deckhouse for Maximizing Cargo Capacity

[15] F.X. Sireta, J. De Lauzon, F. Surmont: HOMER User guide. Bureau Veritas, Paris, France, 2013.

[16] MSC Software: MD Nastran 2010 Dynamic analysis user's guide. Newport Beach, California, USA, 2010.

[17] Bureau Veritas: Hydrostar, User's manual. Paris, France, 2006.

[18] H.I. Im, N. Vladimir, Š. Malenica, D.S. Cho: Hydroelastic response of 19,000 TEU class ultra large container carrier with novel mobile deckhouse for maximizing cargo capacity, International Journal of Naval Architecture and Ocean Engineering, (submitted), 2016.

[19] F.X. Sireta, Q. Derbanne, F. Bigot, Š. Malenica, E. Baudin: Hydroelastic response of a ship structural detail to seakeeping loads using a top-down scheme. Proceedings of the International Conference on Ocean, Offshore and Arctic Engineering - OMAE, ASME, Rio de Janeiro, Brazil, 2012. https://doi.org/10.1115/OMAE2012-83560

[20] Bureau Veritas: Structural Rules for Container Ships, Rule Note NR 625, Paris, France, 2016.

Submitted: $\quad 19.8 .2016$

Accepted: $\quad 02.12 .2016$
Hong-Il Im

Hyundai Heavy Industries Co., Ltd.

Ulsan, Korea

okil@hhi.co.kr

Nikola Vladimir

University of Zagreb, Faculty of

Mechanical Engineering and Naval

Architecture

I. Lučića 5, 10000 Zagreb, Croatia

nikola.vladimir@fsb.hr

Šime Malenica

Bureau Veritas, Research Department

67/71 Boulevard du Châteu,

92200 Neuilly-sur-Seine, France

sime.malenica@bureauveritas.com

Dae Seung Cho

Pusan National University, Dept. of Naval

Architecture and Ocean Engineering

Busan, Korea

daecho@pusan.ac.kr 